\title{
Sugar administration to newly emerged Aedes albopictus males increases their survival probability and mating performance
}

\author{
Romeo Bellini ${ }^{a}$,*, Arianna Puggioli ${ }^{a}$, Fabrizio Balestrino ${ }^{a}$, Paolo Brunelli ${ }^{a}$, Anna Medici ${ }^{a}$, \\ Sandra Urbanelli ${ }^{\mathrm{b}}$, Marco Carrieri ${ }^{\mathrm{a}}$ \\ a Centro Agricoltura Ambiente “G.Nicoli”, IAEA Collaborating Centre, Via Argini Nord 3351, 40014 Crevalcore, Italy \\ b Department of Environmental Biology, University of Rome "La Sapienza", Rome, Italy
}

\section{A R T I C L E I N F O}

\section{Article history:}

Received 11 June 2013

Received in revised form

19 November 2013

Accepted 23 November 2013

Available online $\mathrm{xxx}$

\section{Keywords:}

Sugar

Aedes albopictus

Sterile male

Survival

Mating competitiveness

Sterile insect technique (SIT)

\begin{abstract}
A B S T R A C T
Aedes albopictus male survival in laboratory cages is no more than 4-5 days when kept without any access to sugar indicating their need to feed on a sugar source soon after emergence. We therefore developed a device to administer energetic substances to newly emerged males when released as pupae as part of a sterile insect technique (SIT) programme, made with a polyurethane sponge $4 \mathrm{~cm}$ thick and perforated with holes $2 \mathrm{~cm}$ in diameter. The sponge was imbibed with the required sugar solution and due to its high retention capacity the sugar solution was available for males to feed for at least $48 \mathrm{~h}$. When evaluated in lab cages, comparing adults emerged from the device with sugar solution vs the device with water only (as negative control), about half of the males tested positive for fructose using the Van Handel anthrone test, compared to none of males in the control cage. We then tested the tool in semi-field and in field conditions with different sugar concentrations (10\%,15\%, and $20 \%$ ) and compared results to the controls fed with water only. Males were recaptured by a battery operated manual aspirator at 24 and $48 \mathrm{~h}$ after pupae release. Rather high share $10-25 \%$ of captured males tested positive for fructose in recollections in the vicinity of the control stations, while in the vicinity of the sugar stations around $40-55 \%$ of males were positive, though variability between replicates was large. The sugar positive males in the control test may have been released males that had access to natural sugar sources found close to the release station and/or wild males present in the environment. Only a slight increase in the proportion of positive males was obtained by increasing the sugar concentration in the feeding device from $10 \%$ to $20 \%$. Surprisingly, modification of the device to add a black plastic inverted funnel above the container reduced rather than increased the proportion of fructose positive males collected around the station. No evidence of difference in the capacity of sterile (irradiated with $30 \mathrm{~Gy}$ ) males to take a sugar meal relative to fertile males was observed in field comparison. A clear effect of temperature and relative humidity $(\mathrm{RH})$ on the rate of sugar positive males was observed, with an increase of temperature and a decrease in RH strongly increasing the \% of sugar positive males. In large enclosures we tested the effect of our sugar supplying tool on the mating competitiveness of sterile $v$ s fertile males, which produced an evident favorable effect both on sterile and fertile males.
\end{abstract}

(c) 2013 International Atomic Energy Agency. Published by Elsevier B.V. All rights reserved.

\section{Introduction}

Adult male mosquitoes rely on frequent ingestions of plant produced or insect secreted substances (i.e. aphid honeydew) in order to survive, swarm and possibly to mate (Foster, 1995). Carbohydrates such as fructose, glucose, sucrose, maltose, turanose, melibiose, eriose, melezitose, raffinose and others have been detected in wild collected Aedes albopictus adults by gaschromatography methods (Burkett et al., 1998). Anopheles gambiae

\footnotetext{
"Biology of Male Mosquitoes in Relation to Genetic Control Programmes".

* Corresponding author. Tel.: +3951 873436 .

E-mail address: rbellini@caa.it (R. Bellini).
}

males need to take an energetic meal every night, though female feeding is substantially less frequent, averaging about one sugar feed every four nights (Gary and Foster, 2006). In the field, the energetic costs of swarming for male Anopheles freeborni have been estimated to be about $50 \%$ of a male's available calories per night (Yuval et al., 1994). It is therefore obvious that the capacity of males to find and feed upon sugar sources in nature largely influences their reproductive success, or, in the case of sterile insect technique (SIT) programs, their capacity to induce sterility in the local target population.

The capacity of laboratory reared males to find sugar sources in the wild may be influenced by the selection for phenotypic traits beneficial in an artificial environment, and this may have an important impact on their likelihood of survival when released in the 
field. Domestication may lead to abnormal behaviors in laboratory reared insects (Bartlett, 1984); indeed one study found that after just 9-16 weeks of colonization males Culex tarsalis were unable to compete for wild females (Reisen et al., 1982). The exposure to ionizing radiation commonly used to sterilize mosquitoes for use in the SIT causes somatic damage which may affect ability to perform well in the field (Helinski et al., 2009) though the dose and timing can be adjusted to minimize the negative effects (Balestrino et al., 2010). In the context of mosquito control strategies relying on the release of male adults which must disperse, find and successfully mate with wild females, such as the SIT, it is of crucial importance to formulate optimal larval diets in order to produce sterile males which are highly competitive with wild males. Many studies have been conducted to test various forms of larval diet, both in Ae. albopictus (Yoshioka et al., 2012; Puggioli et al., 2013) as well as other species such as Anopheles arabiensis (Damiens et al., 2012, Hood-Nowotny et al., 2012) and Anopheles stephensi (Khan et al., 2013). Maximising the quality of larval diet could help to compensate for any reduction in male competitiveness caused by the rearing or irradiation processes, as could the provision of energetic substances to adults prior to release (Kassim et al., 2012; Puggioli et al., 2013). Mosquitoes could be released either as adults, in which case an energy rich meal can easily be provided in the time between emergence and release using an approach similar to the one developed for Med fly (Pereira et al., 2011), or as pupae left in the field and allowed to emerge from release devices. In this latter case it might be useful to develop practical systems to administer energetic substances at the release site.

A set of experiments has been conducted in laboratory, semifield and field conditions to obtain information about the feeding behavior of newly emerged Ae. albopictus males. The relative effects of feeding adults with honey vs sugar solution on male and female longevity and female fecundity and fertility were compared. Several tests were also conducted in order to verify the efficiency and optimize the design of a newly developed system for the administration of an energetic source to newly emerged sterile Ae. albopictus males with the aim of increasing their immediate survival and longevity, thus increasing their effectiveness in introducing sterility into the local wild population.

\section{Materials and methods}

\subsection{Mosquito rearing and sugar feeding device}

All Ae. albopictus mosquitoes used for this study were obtained from the pilot mass rearing unit at the Laboratory of the Medical and Veterinary Entomology Department of the Centre for Agriculture and Environment "G. Nicoli". Successive generations of two strains originated from eggs collected in the field in two Italian localities were used to conduct the trials: generations $F_{34}$ and $F_{35}$ of the Pinerolo strain and generations $F_{33}, F_{36}, F_{41}$ and $F_{47}$ of the Rimini strain.

Standard rearing conditions were $28 \pm 1{ }^{\circ} \mathrm{C}, 80 \% \mathrm{RH}$ and a photoperiod of 14:10 (L:D). Adults were kept in Plexiglas cages $(40 \times 40 \times 40 \mathrm{~cm})$ and regularly supplied with a $10 \%$ sucrose solution using a standard laboratory sugar feeder. Females were blood fed with fresh mechanically defibrinated bovine blood, by means of a thermostatically controlled heating apparatus, and eggs were collected on damp filter paper. Larvae were reared in white plastic trays $(41 \times 31 \times 11 \mathrm{~cm})$ containing 31 of deionized water and 4000 first instar larvae $\left(\mathrm{L}_{1}\right)$. Larvae were provided with a standard laboratory diet (Bellini et al., 2007; Medici et al., 2011). Sexing was performed based on size at the pupal stage using a sieving technique (Bellini et al., 2007). Male pupae aged 24-30 h were irradiated

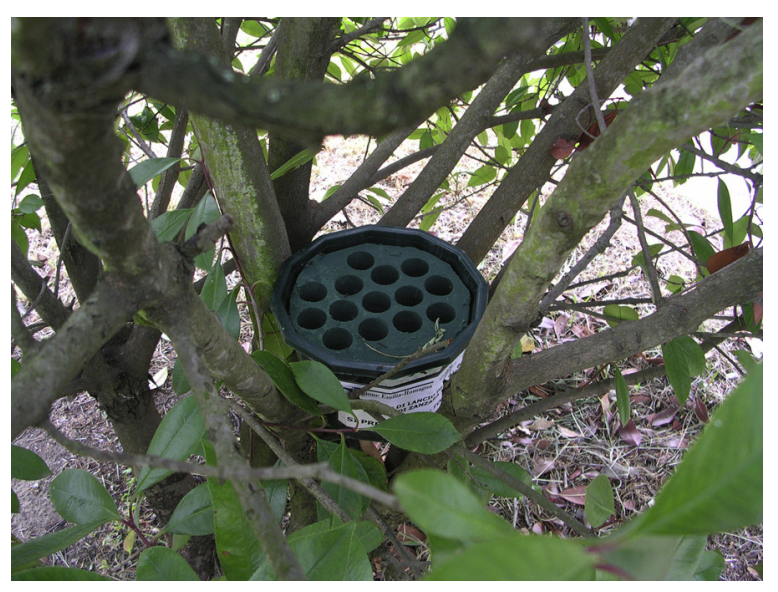

Fig. 1. Device to release sterile male pupae. The device consists of a conical green dark plastic container with a base diameter of $13.5 \mathrm{~cm}$, top diameter of $17.5 \mathrm{~cm}$ and height of $12.5 \mathrm{~cm}$ in which a polyurethane sponge drilled with $2 \mathrm{~cm}$ diameter holes was fitted. The sponge was soaked in the desired sugar solution just before field deployment. The newly emerged males must go through the holes to take flight.

at 30 Gy at the Medical Physics Department of St. Anna Hospital (Ferrara, Italy), just before release (Balestrino et al., 2010).

The sugar feeding devices used as prototypes consisted at the beginning of a $5 \mathrm{~cm}$ thick and $16 \mathrm{~cm}$ diameter foam sponge (Poliform SNC, San Matteo della Decima, Italy, density $30 \mathrm{~kg} / \mathrm{m}^{3}$ ) drilled with seven holes $2 \mathrm{~cm}$ in diameter, and successively of a polyurethane sponge (art. 1009, Greenline International s.r.l. Brendola (VI) Italy), $5 \mathrm{~cm}$ thick and $16 \mathrm{~cm}$ in diameter, in which 11-14 holes each $2 \mathrm{~cm}$ in diameter were drilled. In both cases the device was soaked in a sugar solution and inserted into the inner part of the standard plastic container used for sterile male pupal field release (Bellini et al., 2013a) (Fig. 1). The sponge was soaked in sugar solution and due to its high retention capacity was able to keep the sugar solution available for males to feed for at least $48 \mathrm{~h}$.

\subsection{Fructose intake detection}

Individual mosquitoes were analyzed following the Van Handel method (Van Handel, 1972, 1985) which detects the presence/absence of fructose, or fructose as a component of sucrose. An individual mosquito was placed into a $1.5 \mathrm{ml}$ test tube with $0.5 \mathrm{ml}$ of fructose reagent then crushed with a glass rod. The reagent turning green or blue at room temperature in 10-15 min indicates the presence of fructose. If the reagent didn't change color in an hour, the test was considered to be negative.

\subsection{Laboratory trials}

\subsubsection{Sugar vs honey trial}

A trial was carried out in order to determine the effect of regular sugar (sucrose) solution against honey administration, as an energetic source, on male and female longevity, female fecundity (number of eggs laid) and egg fertility (\% egg hatch).

Fifty female and 50 male pupae (Rimini $F_{33}$ ) were put together into one Plexiglas cage $(40 \times 40 \times 40 \mathrm{~cm})$ and emerged adults fed with a standard laboratory sugar feeder containing a solution of deionized water with $10 \%$ sugar or deionized water with $10 \%$ honey (Multifloral honey, MieliziaBio, Bologna, Italy). Four replicates were performed per treatment.

Mortality of the mosquitoes was checked daily, removing any dead individuals from the cage, for 42 days. Five days after putting males and females together, the mosquitoes were blood fed and the engorged females were counted. Five days after the blood meal the eggs which had been laid were collected, counted and stored in 
a sealed container for embryo maturation. After one week the eggs were submerged in water supplemented with bacterial broth for hatching following the method described in Bellini et al. (2007).

\subsubsection{Newly emerged males' propensity to feed}

In order to investigate the propensity of newly emerged males to feed on sugar solution 50 mechanically sieved male pupae (Pinerolo $\mathrm{F}_{34}$ ), aged $30-40 \mathrm{~h}$, were placed individually into $200 \mathrm{ml}$ plastic containers covered with fine gauze and allowed to emerge. A cotton ball soaked in a $10 \%$ sugar solution was placed on the top of the gauze to allow adult feeding. The eclosion time of each pupa was recorded and at $1,3,7,18,20,22 \mathrm{~h}$ after their emergence a proportion (between 5 and 12) of the adult males were collected and analyzed for fructose content using the Van Handel test.

\subsubsection{Newly emerged males' propensity to feed from the prototype sugar feeding device}

Mechanically sieved male pupae (Pinerolo $\mathrm{F}_{35}$ ), aged $30-40 \mathrm{~h}$, were used to test the propensity of newly emerged males to feed from the prototype sugar feeding device made from foam sponge. Five Plexiglas cages $(40 \times 40 \times 40 \mathrm{~cm})$ were set up with 100 male pupae placed into each in the following containers: the standard container for field release (tronco-conical green dark plastic container with base diameter $13.5 \mathrm{~cm}$, top diameter $17.5 \mathrm{~cm}$, height $12.5 \mathrm{~cm}$ ) without any sugar source as a control; two cages with the standard container for field release provided with the prototype sugar feeding device with a $10 \%$ sugar solution, and two with the standard container for field release with free access to a standard laboratory sugar feeder containing a $10 \%$ sugar solution hanging from one side of the cage.

Every hour from 2.5 to $31.5 \mathrm{~h}$ from the onset of adult emergence all the males emerging in each cage were collected and analyzed for fructose intake using the Van Handel test. The time to emergence was also recorded.

\subsubsection{Effect of the sugar feeding device prototype made by foam sponge on male longevity}

In order to evaluate the effectiveness of a prototype sugar feeding device in providing an energetic source to newly emerged $\mathrm{Ae}$. albopictus males, the longevity of males placed in a Plexiglas cage $(40 \times 40 \times 40 \mathrm{~cm})$ with or without the prototype made by foam sponge was compared.

For this trial mechanically sieved male pupae (Rimini $\mathrm{F}_{33}$ ) aged 30-40 h were used. Six cages were set up: three with 200 male pupae placed inside the standard container for field release without the prototype, and three with 200 male pupae placed inside the standard container for field release provided with the sugar feeding device prototype. Two days after introduction of the pupae into the cage, when all adults were emerged, the prototype was removed and a cotton ball soaked in water only was placed inside each cage. Every day dead individuals were recorded and removed from the cage to calculate longevity.

\subsubsection{Efficacy of the sugar feeding device made by polyurethane sponge}

For this trial a polyurethane sponge was used for the sugar feeding device instead of the foam sponge because, when soaked in the sugar solution, the foam tended to leak, while the polyurethane sponge retained the sugar solution for $48 \mathrm{~h}$ without any leakage. To test the efficacy of this new sugar feeding device mechanically sieved male pupae (Rimini $\mathrm{F}_{36}$ ) aged $30-40 \mathrm{~h}$, were used. Two Plexiglas cages were set up: one with 1000 male pupae placed inside the standard container for field release without an energetic source (as negative control), the other with 1000 male pupae placed inside the standard container for field release provided with the sugar feeding device. Following results obtained in the previous experiment as described at Section 2.3.3, we decided to collect males at $20 \mathrm{~h}$ from the onset of adult emergence, in parallel from each treatment, and analyzed them for fructose intake using the Van Handel test.

\subsection{Semi-field trials}

The effect of sugar administration on the mating competitiveness of fertile and irradiated males was investigated in the summer of 2011 in large net-screened enclosures $(8 \times 5 \times 2.8 \mathrm{~m})$ built in a naturally shaded environment following the protocol described in Bellini et al. (2013b).

The ratio of fertile males/sterile males/virgin females was kept constant at 200/200/100. The sugar feeding device made by polyurethane sponge was provided either to the fertile males or to the sterile males. An inverse funnel was positioned on the top of the sugar feeding station to prevent the other males taking sugar from the device. The virgin females were added as adults 3-6 days after placing the male pupae inside the enclosure.

One blood meal was provided by two technicians entering the enclosures on day 3 after the females' introduction. On the same day four ovitraps (Bamaplast, Massa e Cozzile (Pt), Italy) consisting of a black plastic pot, with a capacity of $400 \mathrm{ml}$, filled to about $2 / 3$ of its height with about $285 \mathrm{ml}$ of dechlorinated water, with three $12.5 \times 2.5 \mathrm{~cm}$ strips of masonite fixed on the internal side for egg deposition, were positioned on the ground in each enclosure. The number of blood fed females was recorded by technicians during blood feeding to enable the mean female fecundity to be calculated from the total number of eggs laid in the ovitraps. All the eggs collected at the end of the trial underwent a standard lab procedure for hatching (Bellini et al., 2007) to estimate the fertility/sterility levels. This experiment was replicated twice, each time in four enclosures.

\subsection{Field trials}

\subsubsection{Study areas}

Release-recapture experiments to investigate the fructose intake of field released Ae. albopictus males were conducted in two urban localities situated in the Po plain, Bologna province in Northern Italy. Boschi di Baricella (latitude $44^{\circ} 41^{\prime} 25^{\prime \prime} \mathrm{N}$, Longitude $\left.11^{\circ} 33^{\prime} 31^{\prime \prime} \mathrm{E}\right)$ has 458 inhabitants and an average population density of 1832 inhabitants $/ \mathrm{km}^{2}$ comprising 168 houses within an area of 16.4 ha. San Giorgio di Piano (latitude $44^{\circ} 38^{\prime} 45^{\prime \prime} \mathrm{N}$, longitude $11^{\circ} 22^{\prime} 35^{\prime \prime} \mathrm{E}$ ) has 6616 inhabitants, an average population density of 4084 inhabitants $/ \mathrm{km}^{2}$ comprising 1132 houses within an area of 141 ha.

Each locality was surrounded by rural areas and included mainly two-story houses, separated by narrow lanes, with many private and some public gardens. These landscape features are representative of most of the small towns in Northern Italy. The presence of Ae. albopictus populations in these areas had been demonstrated by monitoring activities conducted since 2003 (R.B. unpublished data).

\subsubsection{Mosquito release and recapture}

Three release-recapture experiments were conducted between June and September in 2009, 2010 and 2011. In each release around 1000-2000 pupae were placed in each container and allowed to emerge; male recapture sessions were conducted at set time points post release, by means of a battery operated manual aspirator used in an area of 1-2 $\mathrm{m}$ around the station, by agitating the vegetation and collecting flying males for $10 \mathrm{~min}$. The type, number and positioning of release containers, the nature of the male pupae release and the time points at which recapture sessions were conducted varied for each experiment; details are given in Sections 2.5.3-2.5.5. 
In order to evaluate the influence of $\mathrm{RH}$ and temperature on the rate of sugar fed males, the data were corrected using the rate of sugar fed males collected close to stations without the sugar feeding system by means of the Mulla formula (1).

\section{SMS $=($ Ms_S/Ms_T) $/($ Mtest_S/Mtest_T $)$}

SMS: the rate of sugar positive males collected at the sugar feeding stations corrected according to the number of sugar positive sterile males collected at the control stations without sugar; Ms_S: number of sugar positive males collected at the sugar feeding stations; Ms_T: total number of sampled males collected at the sugar feeding stations; Mtest_S: number of sugar positive males collected at the stations without the sugar feeding device; Mtest_T: total number of sampled males collected at the stations without the sugar feeding device.

\subsubsection{Boschi di Baricella, 2009}

Ten release stations were positioned homogenously across the inhabited area at a distance of around $100 \mathrm{~m}$ from each other. Releases of sterile male pupae were conducted weekly from June 19th to August 8th 2009, for a total of eight release-recapture sessions. In the period June 19th-July 4th 2009, eight of the release stations were provided with a $10 \%$ sucrose solution by the polyurethane sponge device (two stations acted as non-sugar treated control), while from July 10th to August 8th 2009, four stations were set with $20 \%$ sucrose solution, four stations with the $10 \%$ sucrose solution and two were used as controls with the polyurethane sponge device soaked in water only without any sugar. Following each release two male recapture sessions were conducted at $20-24 \mathrm{~h}$ and $40-48 \mathrm{~h}$ post release.

\subsubsection{Boschi di Baricella, 2010}

Four release stations were set up in the study area at a distance of about $150 \mathrm{~m}$ from each other. Three release stations were provided with the polyurethane sponge sugar feeding device, while one release station was used as a control without the sugar feeding device being provided. A $20 \%$ sugar solution was tested in the period June 10 th to July 1 st (four releases) with recaptures at 24 and $48 \mathrm{~h}$ post release, while a $10 \%$ sugar solution was tested in the period July 8th to August 24th (nine releases) with recaptures at 24,48 and $72 \mathrm{~h}$ post release, as described above.

One sugar feeding device in each of the three release stations, both at $10 \%$ and $20 \%$ sugar concentration, was modified by adding a reversed black plastic funnel above the container to protect newly emerged adults and to prevent wild males from taking sugar (Fig. 2).

\subsubsection{San Giorgio di Piano, 2011}

Three release stations were set up in the area at a distance of around $400 \mathrm{~m}$ from each other. Two release stations were provided with the polyurethane sponge device soaked in a $15 \%$ sugar solution, while one release station was used as a control without the sugar feeding device.

Fertile or $30 \mathrm{~Gy}$ irradiated male pupae were placed in the two release stations with the sugar feeding device, alternating each time the position of fertile $v s$ irradiated pupae.

Releases were conducted every 2 weeks ( $n=6$ releases) in the period from July 13 th to September 26 th. Recaptures around each release station were conducted at 24 and $48 \mathrm{~h}$ post release ( $n .12$ sessions).

\subsection{Meteorological data}

Data were obtained from two meteorological stations of the Hydro-Meteo-Climate Service of the Emilia-Romagna Regional Agency for Environmental Protection (ARPA-SIMC): located at Mezzolara di Budrio (Long. 11.533793 ${ }^{\circ}$, Lat. $44.571053^{\circ}$ ), and at

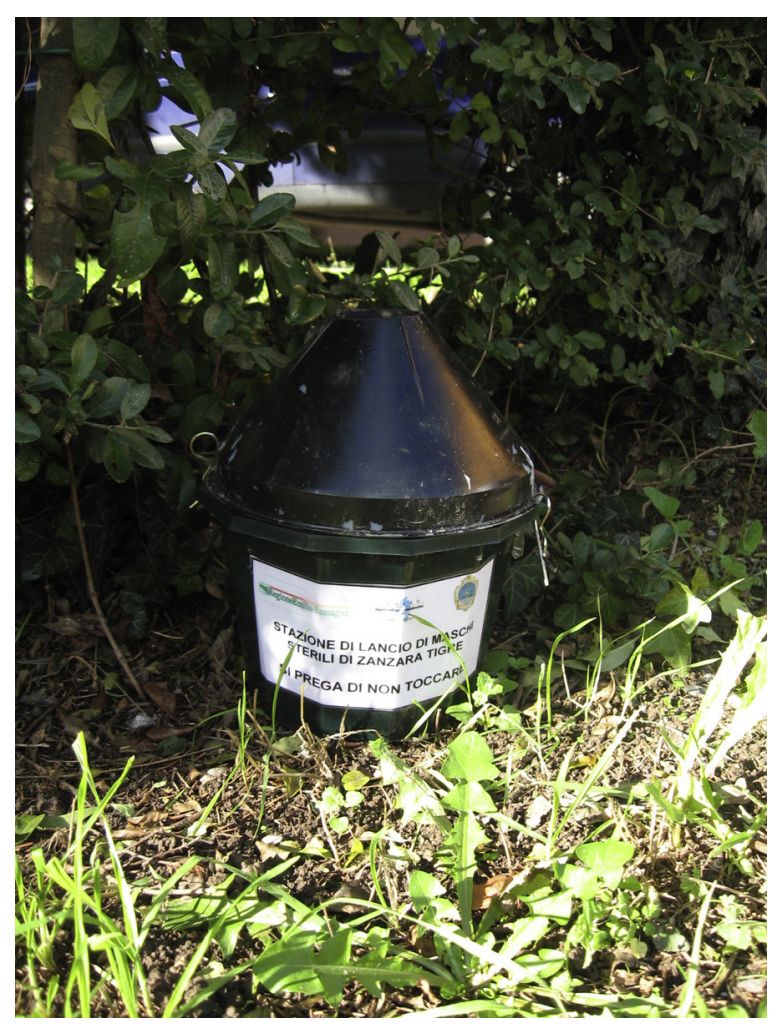

Fig. 2. Male pupae release station adapted with an inverted funnel on the top.

Padulle di Sala Bolognese (Long. $11.290563^{\circ}$, Lat. $44.627752^{\circ}$ ), both in the Bologna province.

\subsection{Statistical analysis}

The Kaplan-Meier survival analysis was used to determine the longevity of males and females fed with sugar or with honey; data sets were compared using the Mantel-Cox log-rank test.

ANOVA was used to determine the effect of availability of the sugar feeding device on the percentage of collected males which were sugar positive. Percentage data were submitted to angular transformation before the analysis. The Newman-Keuls multiple comparison test was used for separation of the means. Pearson product moment correlation $(R)$ was calculated between the weather parameters and the percentage of sugar positive males.

Block ANOVA was used to compare the mating competitiveness of fertile and irradiated males in semi-field trials.

\section{Results}

\subsection{Laboratory trials}

\subsubsection{Sugar vs honey trial}

The longevity of males and females fed with sugar and those fed with honey are reported in Fig. 3. The analysis did not show any significant difference either in male ( $\log$ rank test $=1.34$ and $p=0.18$ ) or female $(\log$ rank test $=1.29$ and $p=0.20)$ longevity between those fed on sugar and those fed on honey. A significant difference (log rank test $=-12.50$ and $p<0.0001)$ was observed between mean male (33.38 \pm 10.07 days) and female (39.88 \pm 6.92 days) longevity.

No significant difference was found in female fecundity between the two feeding treatments $\left(F_{1,6}=1.11\right.$ and $\left.p=0.33\right)$. The mean numbers of eggs laid by females fed with the sugar solution and honey solution were 77.98 (SD 22.92) and 93.22 (SD 17.60), respectively. In addition, no significant difference was found in the fertility 
a
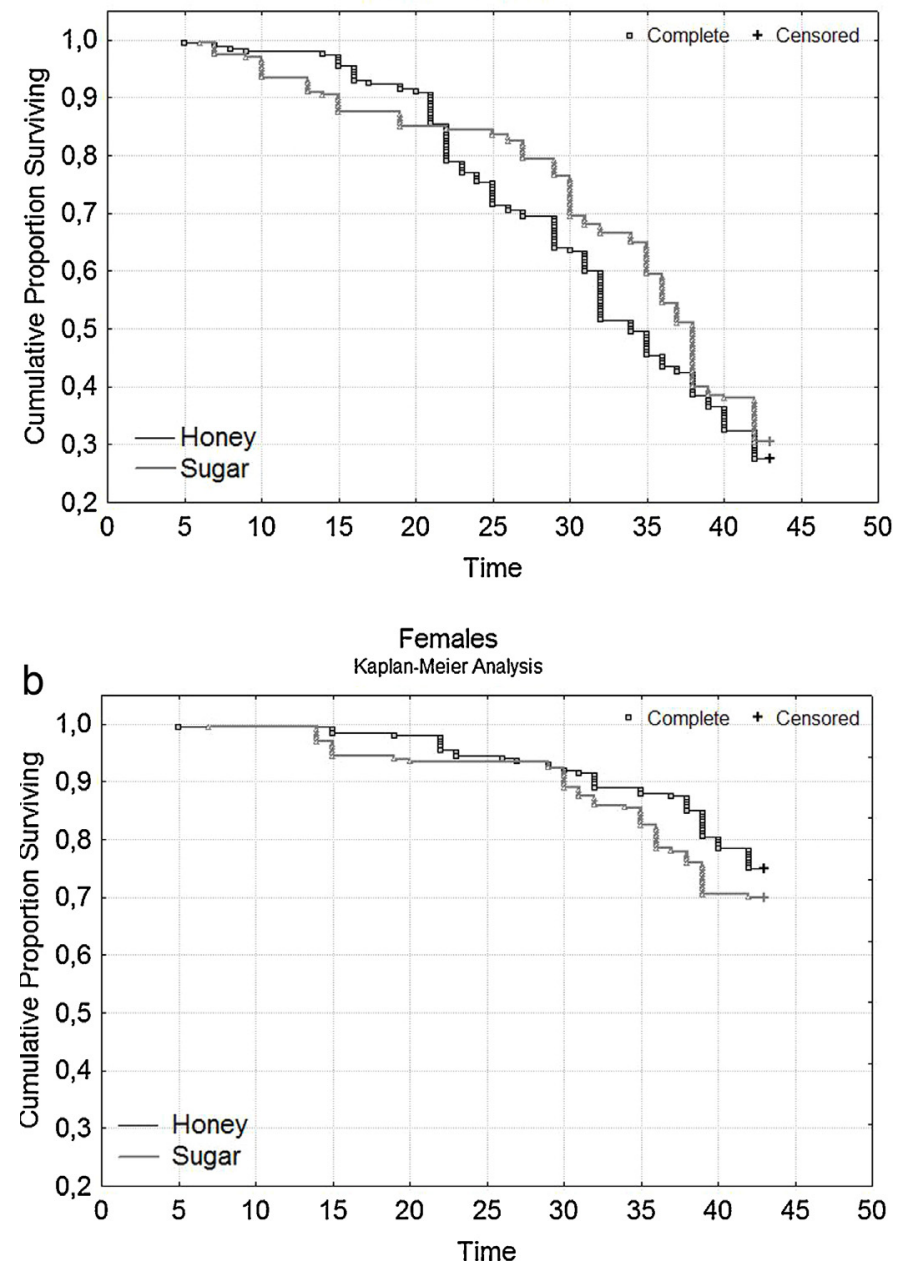

Fig. 3. Male and female curves of longevity (Kaplan-Meier).

(\% egg hatch) between females fed with the two energetic sources $\left(F_{1,6}=0.07, p=0.80\right)$. The mean hatching rate was $87.55 \%$ (SD 9.26) and $89.13 \%$ (SD 7.36) for those fed with sugar and honey solutions, respectively.

\subsubsection{Propensity of newly emerged males to feed}

A positive correlation was observed between the time elapsed since adult emergence and the percentage of males positive to the Van Handel test $\left(R^{2}=0.91 F_{1,4}=40.43, p<0.005\right)$, expressed by the following equation:

Time $=0.17+1.22$ MSwhere MS is the $\%$ of sugar positive males.

The time necessary for $50 \%$ of males to be positive to the Van Handel test $\left(T_{50}\right)$ was $6.05 \mathrm{~h}\left(3.73-9.81 \mathrm{LC}_{95 \%}\right)$, while the time necessary for $90 \%$ of males to be positive $\left(T_{90}\right)$ was $18.68 \mathrm{~h}(10.02-34.82$ $\mathrm{LC}_{95 \%}$ ).

\subsubsection{Propensity of newly emerged males to feed from the} prototype sugar feeding device

Pupal and adult mortality was recorded as $1 \%$ in the control, $4 \%$ in the replicates using the release container with the sugar feeding device and $7.5 \%$ in those using the container without the sugar feeding device; this difference was not significant $\left(F_{2,2}=14.18\right.$ and $p=0.07$ ).

The presence of the prototype sugar feeding device significantly influenced the mean time to emergence from the container $\left(F_{1,2}=31.01\right.$ and $\left.p<0.05\right)$, which occurred at $11.96 \mathrm{~h}$ (SD 0.13) after

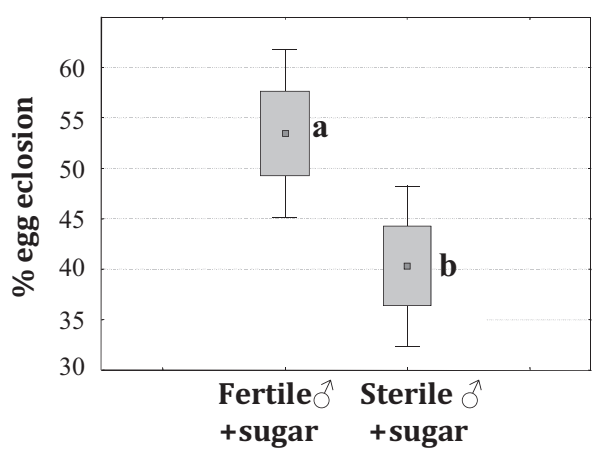

Fig. 4. Influence of the sugar feeding device on the mating competitiveness of Aedes albopictus fertile and sterile males.

deployment of the release devices with the sugar feeding device prototype and $11.23 \mathrm{~h}$ (SD 0.13) without the prototype. The mean percentage of sugar positive males in the cage provided with the prototype was $36.24 \%$ (SD 5.63), not significantly different from the percentage in the cage provided with the standard laboratory sugar feeder $\left(F_{1,2}=3.72\right.$ and $\left.p=0.19\right)$ which resulted in $19.87 \%$ (SD 10.60) of males being positive. Males collected in the control cage where no sugar source was provided all tested negative.

\subsubsection{Effect of the prototype sugar feeding device on male longevity}

Mean longevity was 6.57 days (SD 1.83) for males provided with the prototype sugar feeding device and 5.31 days (SD 0.82) for males without the prototype, a significant difference (test $\log$-rank $=16.62$ and $p<0.001$ ).

\subsubsection{Efficacy of the sugar feeding device made from polyurethane}

The percentage of sugar positive males in the cage provided with the sugar feeding device made from a polyurethane sponge was $46.19 \%$ (SD 17.94), while no positive males was found in the control cage.

\subsection{Semi-field trials}

Pupal and adult mortality in the release containers was $2.50 \%$ (SD 2.83\%) in sterile males provided with sugar, 5.25\% (SD 0.35\%) in fertile males provided with sugar, $4.00 \%$ (SD 0.00\%) in sterile males without access to sugar and $4.00 \%$ (SD $0.71 \%$ ) in fertile males without access to sugar; these differences are not significant $\left(F_{3,4}=1.17\right.$ and $p=0.42$ ).

The mean percentage of eggs hatching, following competition between sterile and fertile males at a $1: 1$ ratio, was $53.47 \%$ (SD $0.08 \%$ ) when fertile males were provided with the sugar feeding device, but only $40.32 \%$ (SD $0.08 \%$ ), corresponding to a capacity to induce sterility (CIS) index value of 1.22 (SD 0.49), when sterile males were provided with the sugar feeding device (Fig. 4), indicating a significant positive effect of the device on male mating competitiveness $\left(F_{1,1}=251.16\right.$ and $\left.p<0.05\right)$.

\subsection{Field trials}

\subsubsection{Boschi di Baricella, 2009}

Pupal and adult mortality in the release containers was $1.03 \%$ (SD 0.36\%) in the control releases, 1.08\% (SD 0.39\%) in the release containers provided with the $10 \%$ sugar solution and $1.24 \%$ (SD $1.27 \%$ ) in the containers provided with the $20 \%$ sugar solution, not significant differences $\left(F_{2,22}=0.19\right.$ and $\left.p=0.83\right)$. 
Table 1

Percentages $( \pm \mathrm{SD}$ ) of sugar positive males field collected close to the (sterile or fertile) male release stations.

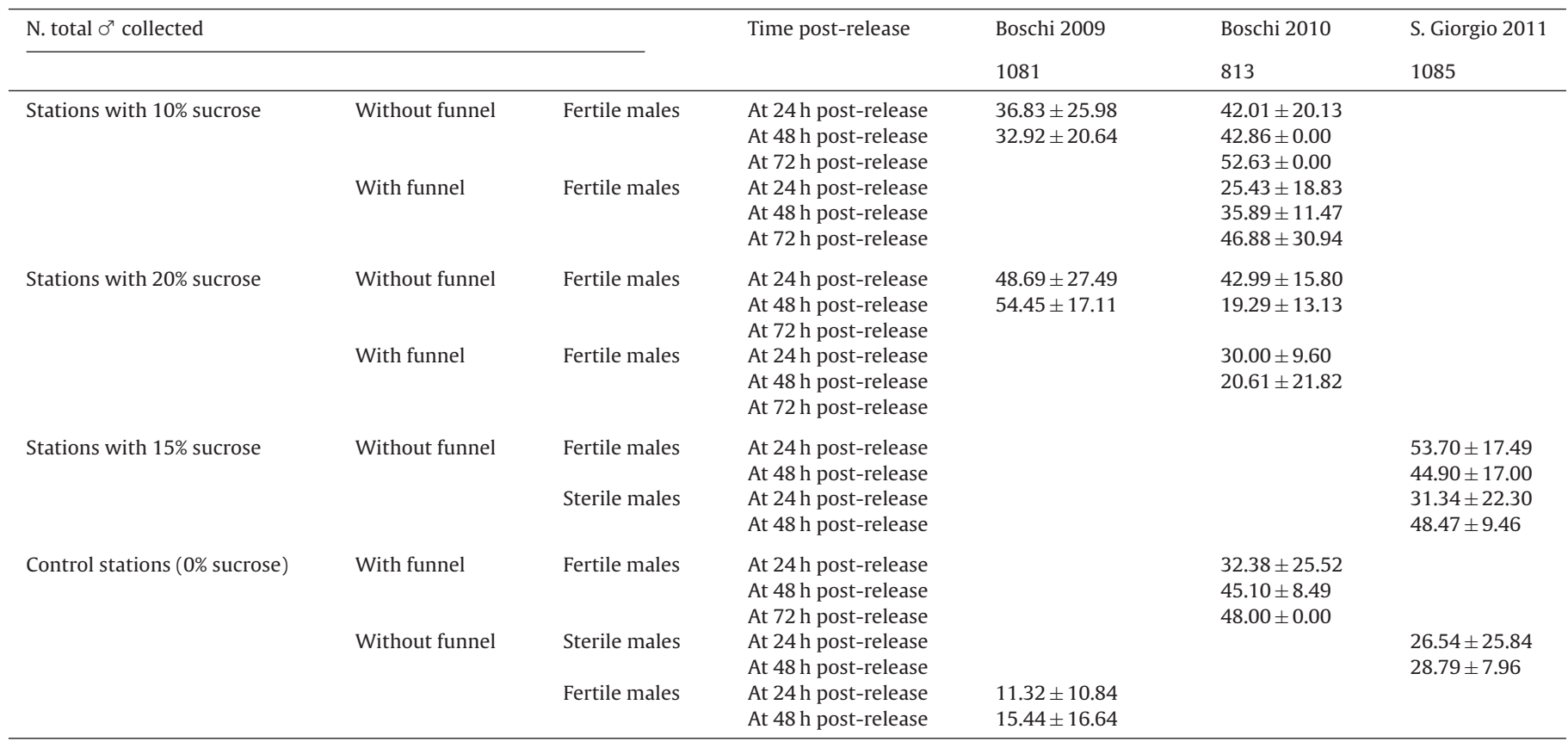

A total of 1081 male adults were collected following the releases and analyzed for fructose intake. The percentage of sugar positive males collected close to sugar treated stations $24 \mathrm{~h}$ after release was significantly higher than the percentage of positive males among those collected around the control stations where sugar was not provided $\left(F_{2,25}=4.52\right.$ and $\left.p<0.03\right)$, while no significant difference was found between the proportion of sugar positive males collected at the stations provided with $10 \%$ sucrose vs stations with $20 \%$ sucrose provision (see Table 1 ). At $48 \mathrm{~h}$ post-release no significant difference was found in the proportion of positive males between treatments $\left(F_{2,8}=3.35\right.$ and $\left.p=0.09\right)$.

A significant correlation was found between the mean temperature $(r=0.83)$ and the maximum RH values $(r=-0.94)$ registered in the study period by the ARPA SIMC meteorological station of Mezzolara di Budrio, and the percentage of positive males collected around the release station provided with the sugar feeding device containing a $10 \%$ sugar solution (see Table 2 ).

\subsubsection{Boschi di Baricella, 2010}

Pupal and adult mortality was $1.04 \%$ (SD 1.01\%) in the control containers, $0.48 \%$ (SD $0.14 \%$ ) in the containers with $10 \%$ sugar solution but without a funnel, 6.18\% (SD 10.88\%) in the containers with $10 \%$ sugar solution and a funnel, $0.75 \%$ (SD 0.42\%) in the containers with $20 \%$ sugar solution but without a funnel, and $2.54 \%$ (SD $3.41 \%$ ) in the containers with $20 \%$ sugar solution and a funnel. No significant differences were found considering sugar solution concentrations (control ( $0 \%$ ), $10 \%$ and $20 \%$ solutions) as the main factor

Table 2

Correlation parameters between rate of sugar positive males collected at sugar treated stations in Boschi di Baricella, 2009 and temperature and RH values.

\begin{tabular}{lrrrllll}
\hline & Mean & \multicolumn{1}{c}{ S.D. } & \multicolumn{1}{c}{$r(X, Y)$} & \multicolumn{1}{l}{$r^{2}$} & \multicolumn{1}{l}{$t$} & $p$ & $N$ \\
\hline RH MIN & 32.57 & 4.96 & -0.789 & 0.622 & -2.87 & 0.035 & 7 \\
RH MEAN & 60.92 & 10.81 & -0.928 & 0.861 & -5.56 & 0.003 & 7 \\
RH MAX & 88.86 & 5.55 & -0.936 & 0.877 & -5.97 & 0.002 & 7 \\
T MIN & 17.33 & 1.52 & 0.666 & 0.444 & 2.00 & 0.102 & 7 \\
T MEAN & 24.79 & 2.99 & 0.834 & 0.696 & 3.38 & 0.020 & 7 \\
T MAX & 32.19 & 3.03 & 0.776 & 0.603 & 2.75 & 0.040 & 7 \\
RAIN & 0.49 & 1.20 & -0.514 & 0.264 & -1.34 & 0.238 & 7 \\
\hline
\end{tabular}

$\left(F_{4.19}=0.93\right.$ and $\left.p=0.47\right)$ or when considering the presence/absence of the funnel $\left(F_{1.16}=1.29\right.$ and $\left.p=0.27\right)$.

A total of 813 male adults were collected and analyzed for fructose intake. The percentage of fructose positive males during the three days of recapture post-release (see Table 1) was not significantly different between those collected near the 10\%, 20\% sugar and control ( $0 \%$ sugar) stations $\left(F_{2,13}=0.73\right.$ and $\left.p>0.50\right)$.

\subsubsection{San Giorgio di Piano, 2011}

Pupal and adult mortality was $7.18 \%$ (SD $8.12 \%$ ) in the control stations without the sugar feeding device, 7.63\% (SD 8.67\%) in the fertile releases with the sugar device and 7.85\% (SD 8.79\%) in the irradiated male releases with the sugar device, not a significant difference $\left(F_{2.14}=0.001\right.$ and $\left.p=0.99\right)$.

A total of 1085 male adults were collected and analyzed for fructose intake. At $24 \mathrm{~h}$ post release the percentage of sugar positive males did not differ between the three types of release station $\left(F_{2.15}=2.39\right.$ and $\left.p=0.126\right)$ while a significant difference was found at $48 \mathrm{~h}$ post release $\left(F_{2.15}=4.33\right.$ and $\left.p=0.033\right)$ between the sugar positive males collected around the two stations provided with sugar and the control station. When combining the total number of positive males captured at 24 and $48 \mathrm{~h}$ post release, a significant difference was also observed $\left(F_{2.15}=5.57\right.$ and $\left.p=0.016\right)$. No significant difference was observed between the ratio of sugar positive males based on whether they were sterile or fertile (determined by means Newman-Keuls multiple comparison test).

The correlation analysis of the \% sugar positive sterile males with temperature and $\mathrm{RH}$ values registered by the ARPA-SIMC meteorological station of Padulle di Sala Bolognese, showed that more of the sterile males released at the control station tended to feed on naturally available sugar sources when the RH increased $(r=0.88$ and $p<0.02)$, while the percentage of positive sterile males decreased when the max temperature increased $(r=-0.79$ and $p=0.06$ ). Analysis of the effect of $\mathrm{RH}$ and temperature on the rate of sugar fed sterile males collected at the sugar feeding stations $v s$ sterile males collected at the control station (SMS) showed a negative correlation with $\mathrm{RH}(r=-0.88$ and $p<0.05)$ and a positive correlation with max temperature $(r=0.02$ and $p<0.01)$ (Fig. 5). 
a
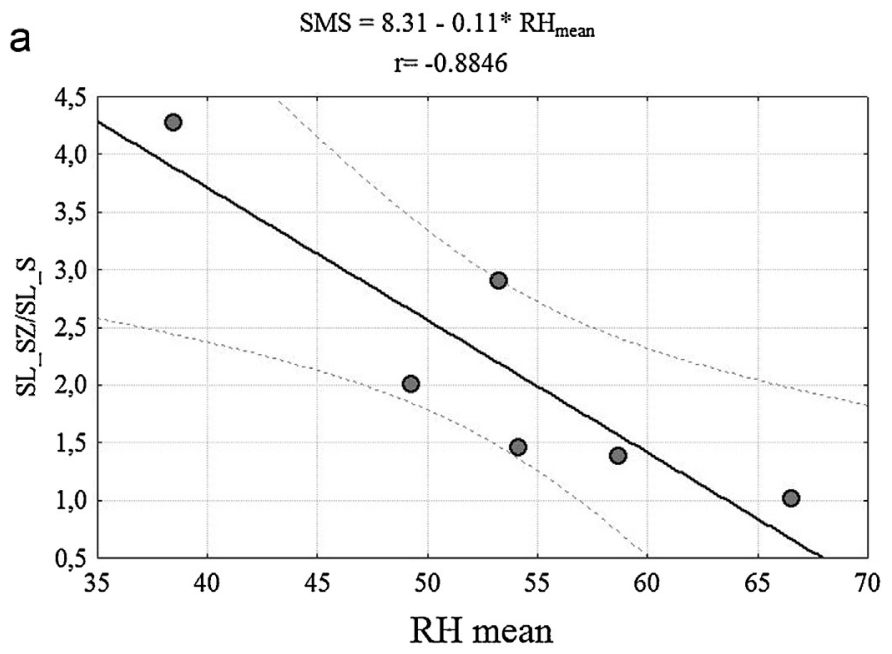

$\mathrm{SMS}=-5.16+0.30 * \mathrm{~T}_{\text {mean }}$

$\mathrm{r}=0.8534$

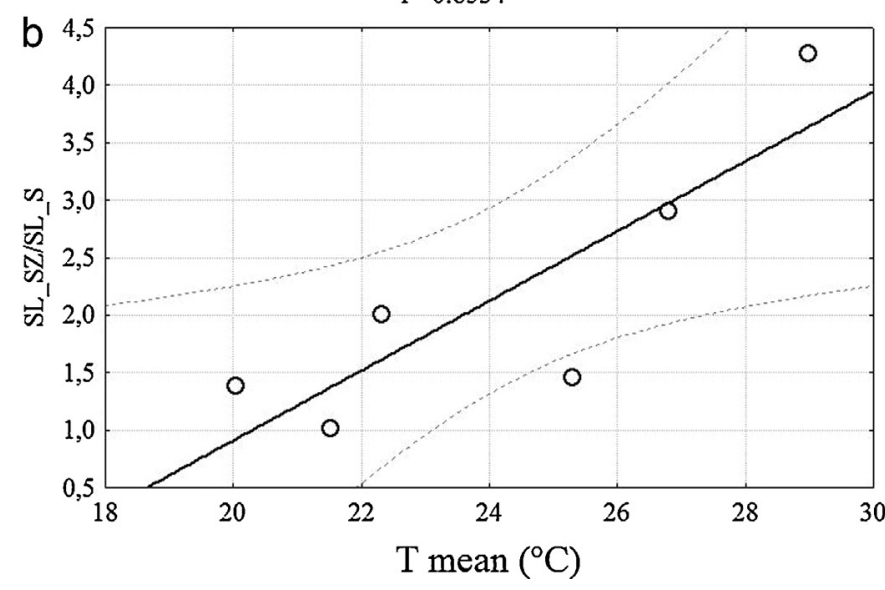

Fig. 5. Correlation lines between the increase in the rate of sugar positive males produced by the sugar feeding device and $\mathrm{RH}$ and temperature values.

\section{Discussion}

The information available on the energy metabolism in mosquitoes is scarce and refers, mainly, to female mosquitoes. Many mosquitoes emerge with diet-dependent carbohydrate reserves (i.e. glycogen) and lipids (i.e. triglycerides) accumulated in the larval stage (Nayar, 1968, 1969; Nayar and Sauerman, 1975; Yuval, 1992; Foster, 1995). Immediately after emergence, many processes that are initiated require significant amounts of energy. In anautogenous mosquitoes these reserves are necessary for the immediate survival and for the first dispersal flight, while in the autogenous species reserves also serve for the maturation of the first gonotrophic cycle. Nayar and Pierce (1977) found that the rate of use of triglycerides in the first $12-24 \mathrm{~h}$ following emergence depends on the amount present: mosquitoes that have a higher accumulated reserve of triglycerides have a usage rate lower than those who emerge with a lower reserve. This phenomenon is similar to that which occurs when the mosquitoes are offered a sugar meal to be used for the survival and synthesis of additional reserves. Van Handel (1965) showed that the rate of depletion of sugar is proportional to the quantity present inside the body of the mosquito: when the percentage of sugar exceeds a certain threshold, its depletion is much slower.

In the laboratory the tests we conducted to compare the effect of sugar vs honey as an energy source found no significant difference in any of the parameters considered, though a non-significantly greater number of eggs were laid by females nourished with honey solution compared to the females nourished with sugar solution. Due to the higher cost of honey (about $7 € / \mathrm{kg}$ ) compared to sugar (about $1 € / \mathrm{kg}$ ) it was decided to use the more common sugar (sucrose) as energetic source both for laboratory mosquito rearing and for the remaining experiments.

In order to design a system to administer sugar (or other nutrient products) to newly emerged males in the field, it is important to establish if the males were inclined to feed on sugar immediately upon or very shortly after emergence. Since a positive correlation was found in the lab between the time elapsed from adult emergence and the percentage of positive males to the Van Handel test for fructose intake, an experiment was carried out to investigate the newly emerged males' propensity to feed from the prototype sugar feeding device (made from foam sponge). No difference was found in the rate of sugar positive males collected in the cages with the prototype $v s$ the cages with the normal lab sugar feeder. Since the males were collected when emerged from the container and flying in the cage, our data suggest that they can easily locate and take the sugar from the dispenser in the cage. The slightly longer emergence time observed in the cages with the prototype vs the cages without the prototype suggest that the males stopped to feed on sugar for a while before flying out. The prototype made with foam sponge was not performing satisfactorily because of its low water retention capacity causing it to suffer intense evaporation when positioned in the field, so the foam was replaced with a polyurethane sponge which had a higher water retention capacity (about 31 times its weight). When tested in the lab, it was observed that almost half of the males fed on the new sugar feeding device, demonstrating its effectiveness.

In another lab experiment we demonstrated that the prototype sugar feeding device had a positive effect on the longevity of males. The effect of the sugar feeding device was also tested in semi-field conditions, by organizing mating competitiveness experiments between sterile and fertile males with and without the sugar feeding device being provided. The tests showed that the sugar feeding device has significant beneficial influence on the mating competitiveness of young males, both fertile and sterile, indicating that the device and release protocol is worth developing further. From our semi-field data it is possible to estimate that the sugar feeding device increases by about $25 \%$ the CIS index value of $30 \mathrm{~Gy}$ irradiated males, reported to be $0.96 \pm 0.62$ in a previous study performed with the same protocol (Bellini et al., 2013b).

In the 2009 field trial no difference in the rate of sugar positive males was found between treatments offered $10 \%$ and $20 \%$ sugar solution concentrations, suggesting that increasing the sugar concentration above $10 \%$ doesn't increase the number of males which feed upon it.

In the 2010 field trial a modified version of the release station fitted with the sugar feeding device was tested. An inverted black plastic funnel was placed on the top of the device in order to increase shade, to perhaps enhance the feeding rate of the newly emerged males and to protect them in their delicate, early adult stage. Unexpectedly the results showed that the presence of the funnel tended to reduce instead of increase the sugar feeding of the males, which is an effect difficult to explain. The inverted funnel was also intended to prevent wild males feeding from the device, but the ratio of wild males feeding from the device without funnel and the effectiveness of the funnel to prevent this were not investigated specifically.

In the 2011 field trial we investigated possible differences in the sugar feeding behavior between fertile and irradiated males, observing no significant difference either at $24 \mathrm{~h}$ or $48 \mathrm{~h}$ postrelease. In this trial the rate of sugar positive sterile males collected at the control stations (who had not been provided with an artificial 
sugar meal) was higher than previously seen indicating the availability of a natural sugar source in the proximity of the station. In general the rate of sugar positive sterile males collected at the control stations was higher at $48 \mathrm{~h}$ post-release in comparison with $24 \mathrm{~h}$ post-release collections, indicating that in our field site the newly emerged males may have found sugar sources close to the release stations.

In both the 2009 and 2011 field trials we found a significant correlation between the mean temperature and $\mathrm{RH}$ and the percentage of sugar positive males collected around the release station without provision of a sugar feeding device, while no such correlation was observed regarding males collected from the release stations provided with the sugar feeding device. In 2011, with $\mathrm{RH}$ values as low as $40 \%$ and mean temperature around $28-29^{\circ} \mathrm{C}$ the proportion of sugar positive males collected at the stations with the sugar device was 4 times the level of males collected around the control stations; when the RH increased, the difference between sugar feeding device and control stations was strongly reduced by an increase in positive males in control stations. As these two parameters (temperature and $\mathrm{RH}$ ) strongly correlate in nature their specific effects are not easily discriminated, but we may assume that young males respond to the perception they have of environmental condition at emergence. The field collected evidence also seems to indicate that during periods of high $\mathrm{RH}$ and low to moderate temperature a greater proportion of the released males may have found a sugar source in nature, close to the release stations, and the provision of supplementary energetic source may be of limited value. Conversely, in the central summer months, when the $\mathrm{RH}$ is lower and the temperature increases, a supplementary energetic source would be highly advantageous to young males.

The methods for field releasing sterile male mosquitoes and the impact they may have on the immediate survival, energy reserves and dispersal capacity of the males, may play an important role in the application of the sterile insect technique. It has previously been suggested (Puggioli et al., 2013) that the provision of a sugar meal prior to release may give an advantage to sterile males in surviving, dispersing and successfully mating in the field. A device which would allow this meal to be provided to adults on emergence from a pupal-release container is here shown to be effective. Moreover the development of an effective system to administer nutrients to sterile male mosquitoes, as well as being valuable in itself, could stimulate the screening and identification of potential substances containing a range of stimulating elements which might improve male mating performance, as was the case in SIT programmes against fruit flies (Pereira et al., 2011).

\section{Acknowledgments}

This work was supported by grants from the Emilia-Romagna Regional Bureau-Public Health Department managed by the Local Health Unit of Cesena, and from the FP7 Infrastructure project "INFRAVEC"Research capacity for the implementation of genetic control of mosquitoes, grant no. 228421. The study has been conducted in the frame of the Joint FAO/IAEA Division of Nuclear Techniques in Food and Agriculture Co-ordinated Research Programme on "Biology of Male Mosquitoes in Relation to Genetic Control Programmes”, CRP G34002.

We also thank the Municipalities of Baricella, Castello d'Argile and San Giorgio di Piano (Bologna) for kindly hosting field experiments.

\section{References}

Balestrino, F., Medici, A., Candini, G., Carrieri, M., Maccagnani, B., Calvitti, M., Maini S., Bellini, R., 2010. Gamma ray dosimetry and mating capacity studies in the laboratory on Aedes albopictus males. J. Med. Entomol. 47, 581-591.

Bartlett, A.C., 1984. Genetic changes during insect domestication. In: King, E.G., Leppla, N.C. (Eds.), Advances and Challenges in Insect Rearing. Agricultural Research Service, New Orleans, LA, pp. 2-8.

Bellini, R., Calvitti, M., Medici, A., Carrieri, M., Celli, G., Maini, S., 2007. Use of the sterile insect technique against Aedes albopictus in Italy: first results of a Pilot Trial. In: Vreysen, M.J.B.A.S., Robinson, J., Hendrichs (Eds.), Area-Wide Control of Insect Pests: From Research to Field Implementation. Springer, Dordrecht, The Netherlands, pp. 505-515.

Bellini, R., Medici, A., Puggioli, A., Balestrino, F., Carrieri, M., 2013a. Pilot field trials with Aedes albopictus irradiated sterile males in Italian urban areas. J. Med. Entomol. 50, 317-325.

Bellini, R., Balestrino, F., Medici, A., Gentile, G., Veronesi, R., Carrieri, M., 2013b. Mating competitiveness of Aedes albopictus radio-sterilized males in large enclosures exposed to natural conditions. J. Med. Entomol. 50, 94-102.

Burkett, D.A., Carlson, D.A., Kline, D.L., 1998. Analysis of composition of sugar meals of wild mosquitoes by gas chromatography. J. Am. Mosq. Control Assoc. 14 373-379.

Damiens, D., Benedict, M.Q., Wille, M., Gilles, J.R., 2012. An inexpensive and effective larval diet for Anopheles arabiensis (Diptera: Culicidae): eat like a horse, a bird or a fish? J. Med. Entomol. 49, 1001-1011.

Foster, W.A., 1995. Mosquito sugar feeding and reproductive energetics. Ann. Rev Entomol. 40, 443-474.

Gary Jr., R.E., Foster, A.A., 2006. Diel timing and frequency of sugar feeding in the mosquito Anopheles gambiae. depending on sex. gonotrophic state and resource availability. Med. Vet. Entomol. 20, 308-316.

Helinski, M.E., Parker, A.G., Knols, B.G., 2009. Radiation biology of mosquitoes. Malar. J. 8 (suppl.2), S6.

Hood-Nowotny, R., Schwarzinger, B., Schwarzinger, C., Soliban, S., Madakacherry, O. Aigner, M., Watzka, M., Gilles, J., 2012. An analysis of diet quality, how it controls fatty acid profiles, isotope signatures and stoichiometry in the malaria mosquito Anopheles arabiensis. PLoS One 7, e45222.

Kassim, N.F., Webb, C.E., Russell, R.C., 2012. The importance of males: larval diet and adult sugar feeding influences reproduction in Culex molestus. J. Am. Mosq. Control Assoc. 28, 312-316.

Khan, I., Farid, A., Zeb, A., 2013. Development of inexpensive and globally available larval diet for rearing Anopheles stephensi (Diptera: Culicidae) mosquitoes. Parasites \& Vectors 6, 90.

Medici, A., Carrieri, M., Scholte, E.-J., Maccagnani, B., Dindo, M.L., Bellini, R., 2011. Studies on Aedes albopictus larval mass-rearing optimization. J. Econ. Entomol. 104, 266-273.

Nayar, J.K., 1968. The biology of Culex nigripalpus Theobald (Diptera: Culicidae), Part 2. Adult characteristic at emergence and adult survival without nourishment. J. Med. Entomol. 5, 203-210.

Nayar, J.K., 1969. Effects of larval and pupal environmental factors on biological status of adults at emergence in Aedes taeniorhynchus (Wiedermann). Bull. Entomol. Res. 58, 811-827.

Nayar, J.K., Sauerman Jr., D.M., 1975. The effects of nutrition on survival and fecundity in Florida mosquitoes. Part 1. Utilization of sugar for survival. J. Med. Entomol. 12, 92-98.

Nayar, J.K., Pierce, P.A., 1977. Utilization of energy reserve during survival after emergence in Florida mosquitoes. J. Med. Entomol. 14 (1), 54-59.

Pereira, R., Yuval, B., Liedo, P., Teal, P.E.A., Shelly, T.E., McInnis, D.O., Hendrichs, J., 2011. Improving sterile male performance in support of programmes integrating the sterile insect technique against fruit flies. J. Appl. Entomol. http://dx.doi.org/10.1111/j.1439-0418.2011.01664.x.

Puggioli, A., Balestrino, F., Damiens, D., Lees, R.S., Soliban, S.M., Madakacherry, O. Dindo, M.L., Bellini, R., Gilles, J.R.L., 2013. Efficiency of three diets for larval development in mass rearing Aedes albopictus (Diptera: Culicidae). J. Med. Entomol. 50 (4), 819-825.

Reisen, W.K., Milby, M.M., Asman, S.M., Bock, M.E., Meyer, R.P., McDonald, P.T. Reeves, W.C., 1982. Attempted suppression of a semi-isolated Culex tarsalis population by the release of irradiated males: a second experiment using males from a recently colonized strain. Mosq. News 42, 565-575.

Van Handel, E., 1965. The obese mosquito. J. Physiol. 181, 478-486.

Van Handel, E., 1972. The detection of nectar in mosquitoes. Mosq. News 32, 458

Van Handel, E., 1985. Rapid determination of glycogen and sugar in mosquitoes. J Am. Mosq. Control Assoc. 1, 299-304.

Yoshioka, M., Couret, J., Kim, F., McMillan, J., Burkot, T.R., Dotson, E.M., Kitron, U., Vazquez-Prokopec, G.M., 2012. Diet and density dependent competition affect larval performance and oviposition site selection in the mosquito species Aedes albopictus (Diptera: Culicidae). Parasites Vectors 5, 225.

Yuval, B., 1992. The other habit- sugar feeding by mosquitoes. Bull. Soc. Vector Ecol. $17,150-156$.

Yuval, B., Holliday-Hanson, M., Washino, R.K., 1994. Energy budget of swarming male mosquitoes. Ecol. Entomol. 19, 74-78. 